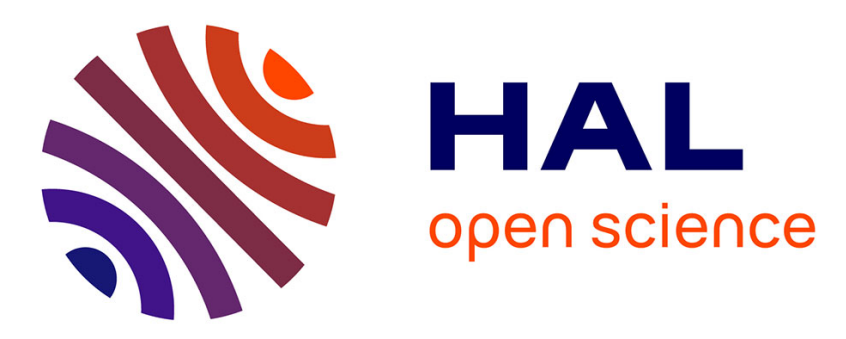

\title{
Field-free one-dimensional alignment of ethylene molecule
}

\author{
A. Rouzée, S. Guérin, Vincent Boudon, B. Lavorel, O. Faucher
}

\section{To cite this version:}

A. Rouzée, S. Guérin, Vincent Boudon, B. Lavorel, O. Faucher. Field-free one-dimensional alignment of ethylene molecule. Physical Review A : Atomic, molecular, and optical physics [1990-2015], 2006, 73, pp.33418. hal-00396965

\section{HAL Id: hal-00396965 https://hal.science/hal-00396965}

Submitted on 19 Jun 2009

HAL is a multi-disciplinary open access archive for the deposit and dissemination of scientific research documents, whether they are published or not. The documents may come from teaching and research institutions in France or abroad, or from public or private research centers.
L'archive ouverte pluridisciplinaire HAL, est destinée au dépôt et à la diffusion de documents scientifiques de niveau recherche, publiés ou non, émanant des établissements d'enseignement et de recherche français ou étrangers, des laboratoires publics ou privés. 


\title{
Field-free one-dimensional alignment of ethylene molecule
}

\author{
A. Rouzée, S. Guérin, V. Boudon, B. Lavorel, and O. Faucher \\ Laboratoire de Physique de l'Université de Bourgogne, \\ UMR CNRS 5027, Boîte Postale 47870, 21078 Dijon Cedex, France
}

(Dated: February 9, 2006)

\begin{abstract}
We report an experimental study of non-adiabatic laser-induced molecular alignment of ethylene $\left(\mathrm{C}_{2} \mathrm{H}_{4}\right)$ using a linearly polarized short laser pulse of moderate intensity. The information about the confinement of the $\mathrm{C}=\mathrm{C}$ bond axis along the direction of the applied electric field is obtained by measuring the depolarization of a second short pulse of weak intensity interacting with the molecules after they have been exposed to the first pulse. The experimental data are compared with the numerical simulation of the Schrödinger equation written for the non-resonant interaction of an asymmetric top rigid rotor with a linearly polarized electric field. The field-free alignment is described by analyzing the time evolution of the angular distribution of the molecular axes together with the expectation value of the relevant squared direction cosine matrix elements.
\end{abstract}

PACS numbers: 33.80.-b, 32.80.Lg, 42.50.Hz, 42.50.Md

\section{INTRODUCTION}

The manipulation of the spatial coordinates of a molecule with the laser field, and in particular the possibility of aligning (or orienting) molecules along chosen directions [1], is a subject that motivates a large interest among the community of physics and chemistry. For the latter, it means an extra degree of freedom in the control of chemical reactions. Adapting the relative orientation of the reactants allows not only to optimize the yield of the reaction but also, and certainly more important, to favor one of its possible channels and therefore to control the branching ratio of the products. For physicists and photo chemists the interest is two-fold. First, understanding the mechanism of alignment and its description in terms of quantum dynamics is important to gain inside the problem of molecules submitted to strong laser fields. Second, most of interactions depend on the angles that make the molecular axis with the direction of oscillation of the field. In an elementary process like single absorption, the excitation of a specific vibrational mode of the molecule depends of which components of the dipole moment are coupled by the field and therefore on the orientation of the molecule with respect to this field. It also applies for molecules exposed to strong field like for instance in high-order harmonic generation $[2,3]$ or in photo-ionization [4]. Controlling the orientation is therefore a mean to optimize laser-molecule interactions and to favor selected outcomes.

Motivated by all the former aspects, a number of theoretical and experimental works [1] have been devoted to the study of laser-induced molecular alignment. They have shown that molecules having a non-isotropic polarizability can be aligned by a pulsed electric field though a non-resonant interaction. The nature of alignment can be cast into two categories. For smooth laser pulses it depends on the ratio of the pulse duration $\tau_{L}$ to the characteristic rotational period $\tau_{\text {rot }}$. In the long pulse limit [5-9], i.e. $\tau_{L} \gg \tau_{\text {rot }}$, the interaction can be considered adiabatic in a sense that at each time of the system evolution the state vector is up to a phase an eigenvector of the full Hamiltonian, corresponding to the eigenvalue continuously connected to the initial one. As a result, the maximum alignment coincides with the maximum of the field. After the maximum, by following adiabatically the slow falling edge of the pulse, the system returns to its initial field-free state and the alignment vanishes. Although adiabatic alignment is efficient in terms of maximum alignment achievable, it is nevertheless restricted to applications that do not suffer from the presence of the concomitant electric field. This limitation is washed out in case of using short pulses with $\tau_{L} \ll \tau_{\text {rot }}[10-14]$. In this limit, the excitation of the molecule is sudden with respect to the classical rotational period and so the molecule is not aligned during the pulse. Nevertheless, the interaction leads to the production of a rotational wave packet in the ground vibronic level of the molecule. The free evolution of this superposition of states leads to recurring alignment and delocalization of the molecule that coincide to the rephasing of the rotational components (i.e., rotational revivals). Compare to the adiabatic regime, where the alignment (or planar delocalization) corresponds to a minimum of the interaction potential for the Hamiltonian, in the short pulse limit the alignment and delocalization alternate in time from one extremum to the other. Alignment corresponds to the minimum and planar delocalization to the maximum, or reversely, depending on the polarization of electric field and the molecule. Some experiments on field-free alignment have been performed in an intermediate regime for which the alignment already appear during the turn off of the laser field $[11,12,15]$. This can be due generally either to the condition $\tau_{L} \sim \tau_{\text {rot }}$ or to field intensity or temperature effects populating highly lying rotational states for which the pulse duration can be comparable to their period of rotation despite the condition $\tau_{L} \ll \tau_{\text {rot }}[10,16]$.

The efficiency of field-free alignment can be improved by making use of pulse tailored with abrupt edges. In this case, the pulse duration appearing in the former relations should be replaced by the time characterizing 
the edges. Assuming that, an asymmetric shaped pulse with a long turn on and a fast turn off will adiabatically align the molecule during the rising time of the pulse, whereas the abrupt falling edge of the pulse will leave the molecule in a broad rotational wave packet, whose recurrence leads to an efficient alignment under field-free conditions [10]. This idea has been demonstrated experimentally in the field-free alignment of linear molecules by creation of "switched" rotational wave packets [17]. Another strategy for the optimization of alignment proposed to use a train of short pulses of specific timing and pulse ratios [18-20]. It was demonstrated experimentally by aligning molecules with use of two time delayed laser pulses [21, 22]. Controlling the alignment and more specifically the time evolution of the molecular axis angular distribution is also an important issue that is currently explored. Based on the idea of controlling the relative phase between the rotational components of the wave packet [23], coherent switching of alignment [24, 25] as well as angular distribution alteration between alignment and planar delocalization have been demonstrated recently [25].

All previously mentioned works concern alignment of linear molecules with linearly polarized laser field. In this case, it is known that the angular potential imposed by the field depends on only one angle $\theta[8,26]$, the polar angle, among the three Euler angles of rotation $(\theta, \chi$, $\phi)$ that describe the orientation of the molecule in the laboratory-fixed frame. This type of interaction leads to one-dimensional alignment of the molecule in a sense that the two other Euler angles, $\phi$ and $\chi$, are not confined by the potential. In a recent work, elliptic laser pulses have been used to make a linear molecule to align alternatively along two different directions in field-free conditions [27]. Symmetric top molecules in a ground vibronic state are quite similar to linear molecules in terms of alignment. In fact, the two equal inertia moments make the symmetry axis of these molecules to align along one laboratory-fixed direction [28]. An asymmetric top molecule, due to the different polarizability of its three axes, can be aligned in three dimensions by the use of an elliptically polarized laser pulse, as it has been first observed in the adiabatic regime with the iodiobenzene molecule [29]. Recently, a strategy based on two time delayed, linearly polarized laser pulses crossed polarized, as been suggested for efficient field-free three dimension alignment of ethylene [30]. So far, only two observations of field-free onedimensional alignment of asymmetric-top molecules have been reported, one in iodiobenzene [15] and the other in iodopentafluorobenzene [31].

The purpose of this paper is to present an experimental and theoretical analysis of the field-free alignment dynamics of ethylene submitted to a linearly polarized laser pulse. In particular, we investigate the intensity dependence of the molecular alignment and show that the molecule tends to rotate preferentially within the molecular plane when it has been aligned by the field. A theoretical model that describes the alignment of an asym- metric top molecule in the short pulse limit and shows the energy distribution of the molecules after interaction with the laser pulse supports the observations.

The following section is devoted to the presentation of the theoretical model and to the discussion of the numerical results. In section III we describe the experimental technique and introduce the quantities that are measured in the experiment. The experimental results are presented in Sec. IV. A comparison between theory and experiment and a discussion of the results are included in the same section. The last section summarizes our work and refers to future prospects.

\section{THEORY AND NUMERICAL RESULTS}

We consider an asymmetric rigid rotor driven by a field

$$
\overrightarrow{\mathcal{E}}(t)=\mathcal{E}_{0}(t) \cos \omega t \vec{e}_{Z}
$$

of amplitude $\mathcal{E}_{0}(t)$ and optical frequency $\omega$, polarized in the $Z$ direction, non resonant with electronic and vibrational states. The Hamiltonian reads [8]

$$
H=H_{0}-\frac{1}{2} \overrightarrow{\mathcal{E}}(t) \cdot \vec{\alpha} \overrightarrow{\mathcal{E}}(t)
$$

with

$$
\begin{aligned}
H_{0}= & B_{x} J_{x}^{2}+B_{y} J_{y}^{2}+B_{z} J_{z}^{2} \\
= & \frac{1}{2}\left(B_{x}+B_{y}\right) J^{2}+\left[B_{z}-\frac{1}{2}\left(B_{x}+B_{y}\right)\right] J_{z}^{2} \\
& +\frac{1}{2}\left(B_{x}-B_{y}\right)\left(J_{x}^{2}-J_{y}^{2}\right)
\end{aligned}
$$

where $B_{\gamma}, \gamma=x, y, z$, is the rotational constant about the $\gamma$ molecular-fixed axis, $J_{\gamma}$ is the component of the angular momentum operator, $J^{2}=J_{x}^{2}+J_{y}^{2}+J_{z}^{2}$, and $\vec{\alpha}$ is the dynamical polarizability tensor, which includes the contribution of the excited electronic states. If we consider frequencies that are low with respect to the excited electronic states, the dynamical polarizability is well approximated by the static ones. In the limit of high frequency with respect to the rotation and far from vibrational resonances, we obtain the effective Hamiltonian $H_{\text {eff }}(t)=H_{0}+V_{\text {int }}$ with

$$
V_{\mathrm{int}}=-\frac{1}{4} \mathcal{E}_{0}^{2}(t) \alpha_{Z Z}
$$

where $\alpha_{Z Z}$ is the diagonal laboratory-fixed component of the polarizability with respect to the field direction. We develop this component as a function of the moleculefixed (diagonal) components using the direction cosines (with respect to $Z$ ) $\cos \theta_{Z \gamma}$ (defined as $Z=\sum_{\gamma} \cos \theta_{Z \gamma} \gamma$ )

$$
\alpha_{Z Z}=\sum_{\gamma} \cos ^{2} \theta_{Z \gamma} \alpha_{\gamma \gamma}
$$


with $\cos ^{2} \theta_{Z x}:=\sin ^{2} \theta \cos ^{2} \chi, \cos ^{2} \theta_{Z y}:=\sin ^{2} \theta \sin ^{2} \chi$, $\cos ^{2} \theta_{Z z}:=\cos ^{2} \theta$ and $\theta, \chi, \phi$ the Euler angles [32]. Using $\sum_{\gamma} \cos ^{2} \theta_{Z \gamma}=1$, we obtain (up to an irrelevant term that does not multiply a direction cosine operator)

$$
V_{\mathrm{int}}=-\frac{1}{4} \mathcal{E}_{0}^{2}(t)\left(\Delta \alpha_{x y} \cos ^{2} \theta_{Z x}+\Delta \alpha_{z y} \cos ^{2} \theta_{Z z}\right)
$$

with $\Delta \alpha_{\gamma \gamma^{\prime}}=\alpha_{\gamma \gamma}-\alpha_{\gamma^{\prime} \gamma^{\prime}}$. For a near-prolate rotor like ethylene we choose the usual $I^{r}$ [33] orientation for which the rotational constants $B_{y} \equiv C \lesssim B_{x} \equiv B<B_{z} \equiv A$ are respectively associated to the axis $c, b$, and $a$, with the polarizabilities $\alpha_{y y} \lesssim \alpha_{x x}<\alpha_{z z}$. The potential (6) of a near-prolate shows a minimum when $\theta_{Z z}=0, \pi$, a maximum when $\theta_{Z y}=0, \pi$, and a saddle point when $\theta_{Z x}=0, \pi$, where we denote $\theta_{Z \gamma}$ the angle between $Z$ and $\gamma$ (see Fig. 1). Thus in the presence of the field, only the $z$-axis of a near-prolate molecule can be predominantly aligned along the field.

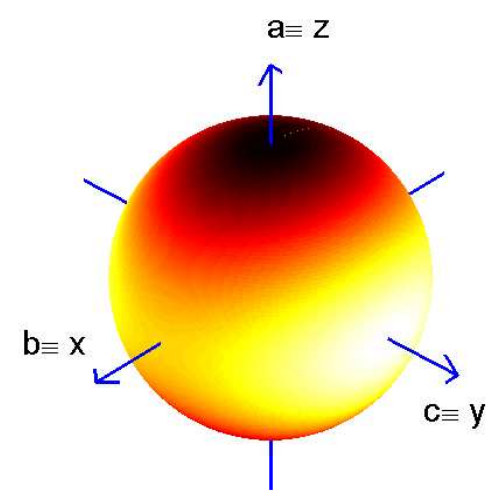

FIG. 1: (Color online) Schematic representation of the potential (6) of a near-prolate molecule on the sphere of coordinates $\theta, \chi, r=1$, where the lowest values of energy are darker. This can be interpreted as the potential in the molecular frame as a function of the polarization axis of spherical coordinates $(\theta, \chi)$.

We use a laser pulse of short duration (denoted $\tau_{L}$ ) that can be treated in the sudden approximation, i.e. when $\tau_{L} \ll \hbar / \max _{\gamma} B_{\gamma}, \gamma=x, y, z$, whose effect is characterized in this case by the dimensionless parameters $\xi_{\gamma y}=\frac{\Delta \alpha_{\gamma y}}{4 \hbar} \int d t \mathcal{E}_{0}^{2}(t), \gamma=x, z$. The selection rules for such a model are $\Delta J=0, \pm 1, \pm 2, \Delta K=0, \pm 2$, $\Delta M=0$, with $K,|K| \leq J(M,|M| \leq J)$ the projection of the angular momentum along the $z$-axis $(Z$ axis). For an asymmetric molecule, $K$ is not a good quantum number, and we should consider instead for a $J$ block the quantum number $\tau:=K_{a}-K_{c}$ with the values $K_{c}=J, J, J-1, J-1, \cdots, 1,1,0$ each respectively associated to $K_{a}=0,1,1, \cdots, J-1, J-1, J, J$ (i.e. the inverse sequence of $K_{c}$ ), where $K_{\gamma}, \gamma=a, c$, is the projection of the angular momentum along the $\Gamma$ molecular axis defined by continuity in the limit of a symmetric-top [33]. The energy $E_{J, \tau}$ increases in a $J$-block as a function of $\tau$. For the Raman processes that we consider here, the transitions preserve the parity of $K_{a}$ and $K_{c}$.
In the case of $\mathrm{C}_{2} \mathrm{H}_{4}, B_{x} \equiv B=1.001 \mathrm{~cm}^{-1} \sim B_{y} \equiv$ $C=0.828 \mathrm{~cm}^{-1}$, and $B_{z} \equiv A=4.864 \mathrm{~cm}^{-1}$ [34], with polarizabilities $\alpha_{x x}=3.682 \AA^{3}, \alpha_{y y}=3.251 \AA^{3}$, and $\alpha_{z z}=5.022 \AA^{3}[35]$. The $z$-axis is along the $\mathrm{C}=\mathrm{C}$ bond and the $x$-axis is orthogonal to the $\mathrm{C}=\mathrm{C}$ bond in the plane of the molecule (see Fig. 2). The spin degeneracy in the ground electronic state gives a specific initial statistical ensemble of the $J$ values: if $K_{a}$ and $K_{c}$ are both even, the spin degeneracy factor is $g_{J, \tau}=7$, otherwise $g_{J, \tau}=3$.

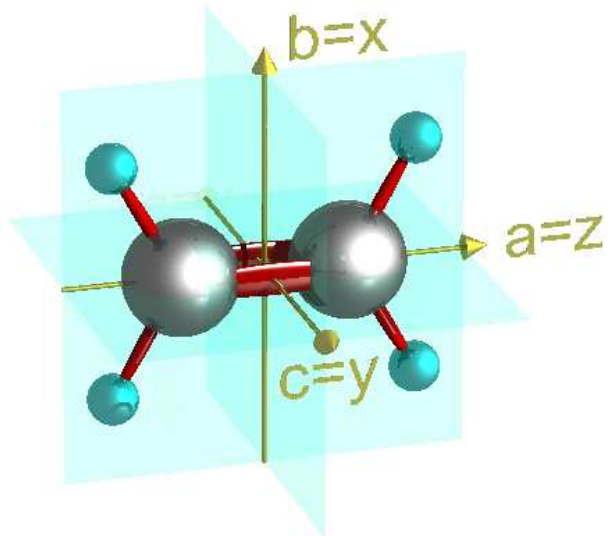

FIG. 2: (Color online) Ethylene molecule depicted with the molecular fixed frame axis $x, y, z$.

We will consider the expectation values of the direction cosines with respect to the direction of the field $Z$ weighted by the Boltzmann thermal distribution:

$$
\left\langle\cos ^{2} \theta_{Z \gamma}\right\rangle(t)=\frac{1}{Z} \sum_{J, \tau} g_{J, \tau} e^{-\frac{E_{J, \tau}}{k T}} \sum_{|M| \leq J}\left\langle\cos ^{2} \theta_{Z \gamma}\right\rangle_{J, \tau, M}(t)
$$

with $Z=\sum_{J, \tau}(2 J+1) g_{J, \tau} e^{-E_{J, \tau} /(k T)}, k$ the Boltzmann constant, and $T$ the temperature. $\left\langle\cos ^{2} \theta_{Z \gamma}\right\rangle_{J, \tau, M}(t) \equiv$ $\left\langle\psi_{J, \tau, M}(t)\left|\cos ^{2} \theta_{Z \gamma}\right| \psi_{J, \tau, M}(t)\right\rangle$ is the expectation value calculated with the state $\left|\psi_{J, \tau, M}(t)\right\rangle$ obtained from the Schrödinger equation with the initial state $|J, \tau, M\rangle$.

The temporal evolution of $\cos ^{2} \theta_{Z z}$ [second term in the right hand side of Eq. (6)] that characterizes the alignment of the $\mathrm{C}=\mathrm{C}$ bond of ethylene ( $z$-axis) along the direction of polarization of the laser field ( $Z$-axis) is shown in Fig. 3 for two laser intensities. The depicted rotational revivals are produced during the free evolution of the system after extinction of the laser field. The molecules are aligned with the $\mathrm{C}=\mathrm{C}$ bond along $Z$ when $\left\langle\cos ^{2} \theta_{Z z}\right\rangle>1 / 3$, whereas the $\mathrm{C}=\mathrm{C}$ bond is delocalized in a plane perpendicular to the $Z$-axis when $\left\langle\cos ^{2} \theta_{Z z}\right\rangle<1 / 3$. As the intensity increases, alignment and planar delocalization improve together with the degree of permanent alignment $[11,36]$ indicated by the presence of an elevated base line $\left(\left\langle\cos ^{2} \theta_{Z z}\right\rangle \gtrsim 1 / 3\right)$. The temporal structure of the revivals is more complicated compared to a linear molecule [14] or, more generally speaking, to a symmetric-top molecule. This is due to the projection $K$ of the angular momentum along 


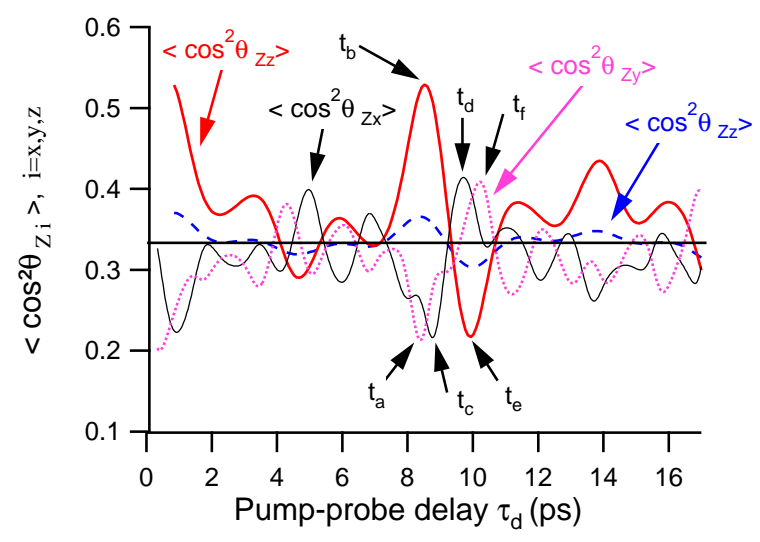

FIG. 3: (Color online) Expectation value of $\cos ^{2} \theta_{Z z}$ calculated in ethylene for a laser peak intensities $I=5 \times$ $10^{12} \mathrm{~W} \cdot \mathrm{cm}^{-2}$ (dashed line) and $30 \times 10^{12} \mathrm{~W} \cdot \mathrm{cm}^{-2}$ (full line). Expectation value of $\cos ^{2} \theta_{Z x}$ (thin line) and $\cos ^{2} \theta_{Z y}$ (thin doted line) for $I=30 \times 10^{12}$ W.cm ${ }^{-2}$. Simulations are performed for a thermal distribution at $5 \mathrm{~K}$. The horizontal line is the expectation value obtained for randomly oriented molecules.

the symmetry $z$-axis that is not conserved during the rotation of an asymmetric top molecule. In the latter, a larger number of rotational states of different periodicity can be populated by the field resulting in rotational wave packets that show more frequency beats. Furthermore, because of the lack of regularity in the rotational-level structure, the rotational components of the wavepacket do not fully rephase after excitation and hence the alignment is not periodic. This stands when the rigid rotor approximation is considered as well. An other consequence arising from the temporal dispersion of the wave packet is the lower efficiency and the rapid decrease of the field-free alignment of the asymmetric top molecule.

The direction cosine $\cos ^{2} \theta_{Z x}$ introduced in the first term of the right hand side of Eq. (6) tells about the alignment of the molecular $x$-axis with respect to the field direction. As well, $\cos ^{2} \theta_{Z y}$ describes the alignment of the molecular $y$-axis. The associated expectation values are represented in Fig. 3. As it is shown, additionally to the $z$-axis, the laser field produces also a fast alternation of alignment and planar delocalization of the $x$ - and $y$-axis, successively. Nevertheless, the effect is less pronounced on these axes because of their reduced polarizability compared to the symmetry $z$-axis. In the $|J, K, M\rangle$ basis of the symmetric top molecule, where the evaluation of the direction cosines is straightforward, one can show that $\left\langle\cos ^{2} \theta_{Z z}\right\rangle$ only involves rotational states with the same $K, \Delta K=0$, with $\Delta J=0, \pm 1, \pm 2$, whereas $\left\langle\cos ^{2} \theta_{Z x, y}\right\rangle$ includes as well $\Delta K= \pm 2$ components. In the limit of a near symmetric top molecule, it explains why the frequency beat is higher for the two last expectation values compared with the former. Futhermore, since a linearly polarized field conserves $K$ in a symmetric top molecule, the difference between the maximum ampli- tudes $\max _{t}\left\langle\cos ^{2} \theta_{Z z}\right\rangle-\max _{t}\left\langle\cos ^{2} \theta_{Z x, y}\right\rangle$ informs about the degree of asymmetry of the polarizability tensor of the molecule.
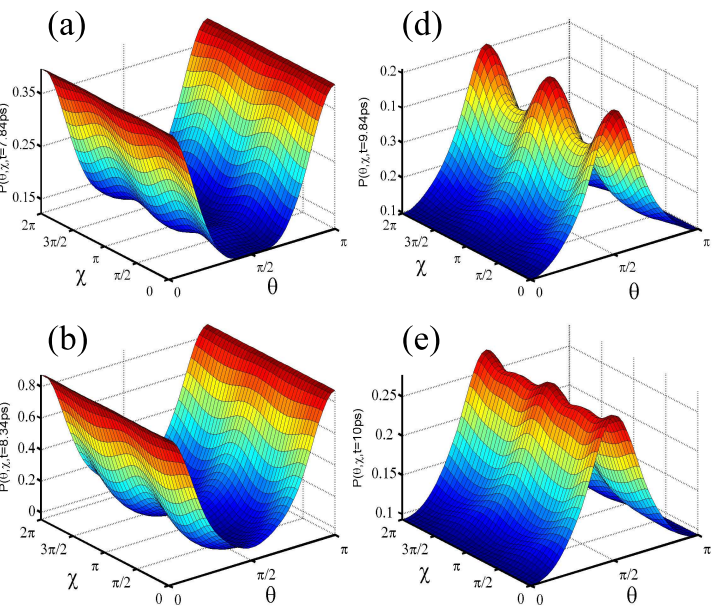

(e)
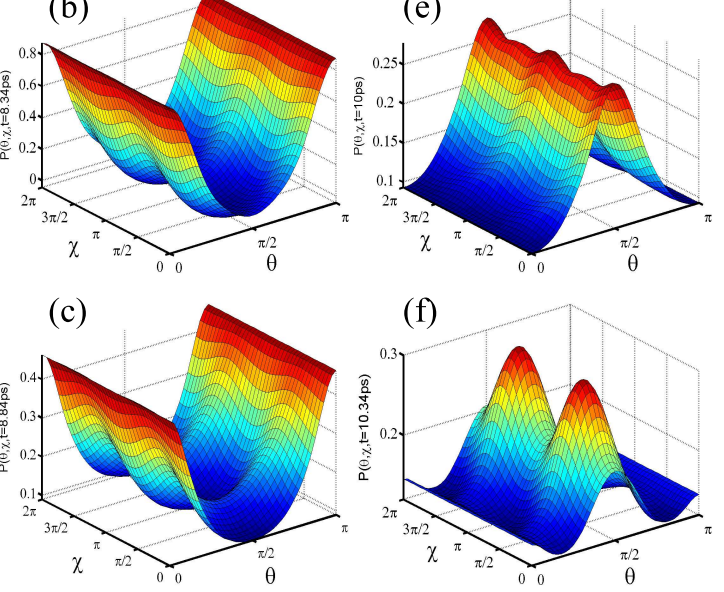

(f)

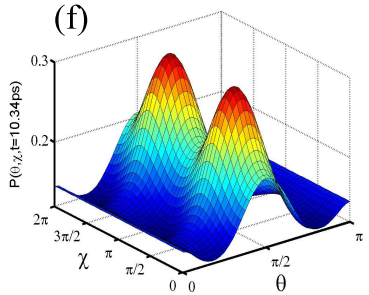

FIG. 4: (Color online) Angular distributions $P(\theta, \chi)$ calculated around the first transient of (a-c) alignment and (d-f) planar delocalization of the molecular $z$-axis at times $t_{j}$ indicated in Fig. 3. (a) and (f) : minimum $\left(t_{a}\right)$ and maximum $\left(t_{f}\right)$ value of $\cos ^{2} \theta_{Z y}=\sin ^{2} \theta \sin ^{2} \chi$, respectively. (b) and (e) : maximum $\left(t_{b}\right)$ and minimum $\left(t_{e}\right)$ of $\cos ^{2} \theta_{Z z}=\cos ^{2} \theta$. (c) and $(\mathrm{d})$ : minimum $\left(t_{c}\right)$ and maximum $\left(t_{d}\right)$ value of $\cos ^{2} \theta_{Z x}=\sin ^{2} \theta \cos ^{2} \chi$.

To get a better insight about the field-free alignment of near asymmetric top molecules, it is convenient to observe the angular distribution of ethylene (Fig. 4) at times that correspond to extrema of each direction cosine, near around the first alignment transient of the molecular $z$ axis, as well as around its first planar delocalization. We see from Fig. 4 (a-c) that when the $z$-axis is aligned in the field direction $(\theta=0, \pi)$, then the two other molecular $x$ and $y$-axes are almost delocalized. It is noticed that full delocalization can only be reached in the limit $\theta \rightarrow 0, \pi$, otherwise the linear polarization tends to align one or the other axis. In the presence of the field, there is a small localization of the $\chi$ angle around $0, \pi$ that corresponds in fact to a minimization of the angle $\theta_{Z x}$ (maximization of $\left.\left\langle\cos ^{2} \theta_{Z x}\right\rangle\right)$ between $Z$ and $x$, the intermediate principal axis of polarizability. This means that with the field on, the molecule is preferentially aligned with the $Z$-axis close to its plane ( $x-z$ plane) [15]. It corresponds to the presence of a saddle point $\left(\theta_{Z x}=0, \pi\right)$ in the potential of Fig. 1. When the $z$-axis is delocalized in the plane 
perpendicular to the field we observe that the two other axis are successively aligned along the field, with first the $x$-axis [Fig. $4(\mathrm{~d}), \chi=0, \pi$ ] and just after with the $y$-axis [Fig. 4 (f)], $\chi=\pi / 2$ ]. In between [Fig. 4 (e)], we observe a superposition of the two $x$ and $y$ axes aligned along the same $Z$-axis $(\chi=0 ; \pi, \pi / 2)$. It is noticed, that the alignment of the $x$-axis is above the $y$-axis one, since the polarizability of the latter is the smallest.

\section{EXPERIMENTAL TECHNIQUE}

Field-free alignment produced by a short pump pulse of moderate intensity is observed by measuring transient birefringence. In this technique, the degree of alignment is probed by using a second weak pulse that interacts with the molecules after they have been exposed to the pump pulse. The anisotropy of the angular distribution of the molecular axes results in the depolarization of the probe that can be monitored versus time by changing the delay between the two pulses. One advantage of this optical technique is that the alignment produced by the pump pulse is preserved as it is probed. In the case of linear molecules, the birefringence signal is proportional to $\left(\left\langle\cos ^{2} \theta\right\rangle-\frac{1}{3}\right)^{2}[14]$, where $\theta$ is the angle between the molecular axis and the polarization direction of the pump electric field and $\frac{1}{3}$ is the isotropic value of $\left\langle\cos ^{2} \theta\right\rangle$. For an asymmetric top molecule, we will show that the signal depends on $\left\langle\cos ^{2} \theta\right\rangle$ but presents also a dependence with $\chi$, the angle of rotation around a major principal axis of polarizability.

The pump and probe pulses are delivered by a Ti:Sapphire laser chirped pulse amplified system operating at $1 \mathrm{kHz}$. The pulse duration is about $100 \mathrm{fs}$ and the wavelength is centered at $800 \mathrm{~nm}$. The scheme of the experiment is presented in detail in Ref. [37]. We only recall here its salient features. The pump and probe are linearly polarized at $45^{\circ}$ to each other. In order to change the intensity of the pump, we use a half-wave plate combined with a polarizer. A delay line composed of a corner cube mounted on a motorized linear stage is placed on the probe path in order to adjust the temporal delay between the pump and probe pulse. Both beams are focused with a $300 \mathrm{~mm}$ focal length lens and cross each other at a small angle $\left(\sim 2^{\circ}\right)$ in a cell filled with $\mathrm{C}_{2} \mathrm{H}_{4}$. The measured waist is $40 \mu \mathrm{m}$ and $60 \mu \mathrm{m}$ for the pump and probe, respectively. After the cell, the pump is blocked by a beam stop and the depolarization of the probe is detected through a polarizer set at $90^{\circ}$ with respect to its initial polarization direction. The outgoing signal field is then collected with a photomultiplier, sampled by a boxcar integrator, and sent to a computer that is also used to control the delay line. The interaction of the two pulses takes place in a $0.2 \mathrm{~m}$-long static cell at room temperature and under pressure of 0.5 bar, which is a compromise to ensure a good signal-to-noise ratio and to minimize the decay of the signal due to collisions.

In the limit of small birefringence, the signal is propor- tional to the convolution of the probe intensity envelope $\mathcal{I}_{p r}(t) \propto \mathcal{E}_{0, p r}^{2}(t)$ and $\Delta n(t)^{2}$, with $\Delta n(t)=n_{\|}(t)-n_{\perp}(t)$ the difference of refractive indices between the parallel $(\|)$ and any perpendicular $(\perp)$ direction of the pump polarization axis:

$$
\mathcal{S}\left(\tau_{d}\right) \propto \int_{-\infty}^{+\infty} d \tau_{d} \mathcal{I}_{p r}\left(t-\tau_{d}\right) \Delta n(t)^{2}
$$

with $\tau_{d}$ the temporal delay between the pump and probe pulses. For asymmetric molecules it can be shown that the difference of refractive indices depends on the angles $\theta$ and $\chi$

$$
\begin{aligned}
\Delta n(t)= & \frac{3 N}{4 n \epsilon_{0}}\left\{\Delta \alpha_{z x}\left(\left\langle\cos ^{2} \theta\right\rangle-\frac{1}{3}\right)\right. \\
& \left.+\Delta \alpha_{y x}\left(\left\langle\sin ^{2} \theta \sin ^{2} \chi\right\rangle-\frac{1}{3}\right)\right\}
\end{aligned}
$$

with $n$ the average value of the refractive index at the probe frequency, $N$ the number density, $\epsilon_{0}$ the dielectric constant of vacuum, and where higher-order polarizabilities have been neglected. For a near symmetric top molecule like ethylene, we have $\left|\Delta \alpha_{z x}\right|>\left|\Delta \alpha_{y x}\right|$ and therefore the birefringence reflects mainly the alignment of the major polarizability $z$-axis (the $\mathrm{C}=\mathrm{C}$ bound axis, in ethylene).

\section{RESULTS AND DISCUSSION}

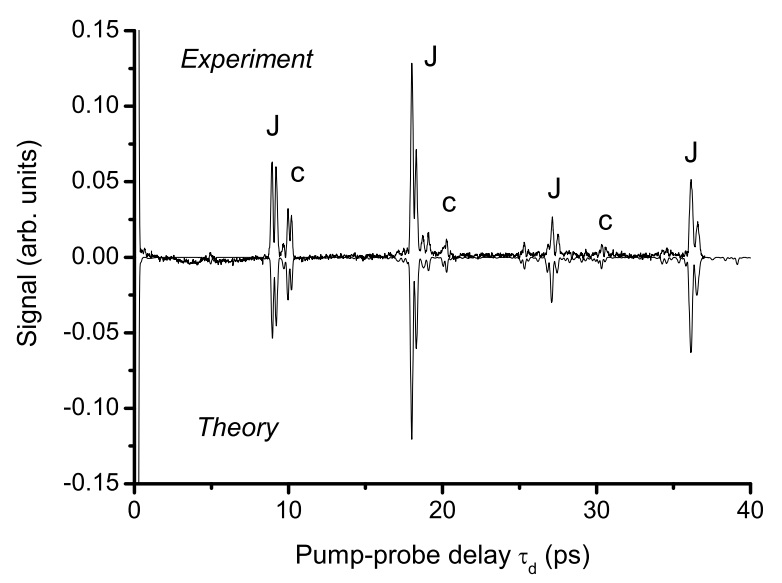

FIG. 5: Probe signal recorded in ethylene at room temperature for a low intensity $\mathcal{I}_{p}=2 \times 10^{12} \mathrm{~W} . \mathrm{cm}^{-2}$ of the pump laser. $J$ and $C$-type transients are indicated. The numerical calculation is shown reversed.

Figure 5 shows the birefringence signal observed after the ethylene molecules have interacted with the alignment pulse (pump). The theoretical curve is obtained from the numerical simulation of Eq. (9). The intensity 
of the pump pulse is kept low so as the alignment remains small $\left\langle\cos ^{2} \theta\right\rangle \simeq 1 / 3$. In this regime, the results can be described in terms of rotational transients as in standard ultrafast rotational coherence spectroscopy [38]. Therein, the temporal transients arise from frequency beatings between the excited rotational transitions, since in this case the distribution of populations is conserved by the weak laser field (perturbative regime). Using this approach, we can identified from Fig. 5 two different types of transients called $J$-type and $C$-type. The former is characterized by a pseudo-period $T_{J}=1 / 2(B+C)=9.112 \mathrm{ps}$ and the latter by $T_{C}=1 / 4 C=10.06 \mathrm{ps}$. For near symmetric top molecules subjected to a low field (i.e., in a weak alignment regime), the $J$-type transients are the most pronounced, as confirmed here for ethylene. They are also the only type observed in pure rotational spectroscopy of symmetric top molecules. The $C$-type transients result from the asymmetry of the molecules and describe a rotation of the molecule about the molecular $c$-axis indicated in Fig. 2. They are produced through $|\Delta J|=\left|\Delta K_{c}\right|=2$ transitions that mainly involve levels belonging to the lowest part of the rotational spectrum : $|J, \tau \approx-J, M\rangle$. The $A$-type transients with a period $T_{A}=1 / 4 A=1.713 \mathrm{ps}$ are not observed in the present study. They principally originate from the upper part of the rotational spectrum $|J, \tau \approx J, M\rangle$ through $|\Delta J|=\left|\Delta K_{a}\right|=2$ transitions. For ethylene at room temperature, this part is weakly populated compared to the lower part of the spectrum and so the $A$-type transients are much smaller compared to the $C$-type transients [39].

The intensity alternation observed one every second revival is due to selection rules. The $\Delta J= \pm 1$ transitions produce $J$ - and $C$-type transients with a period that is twice compare to the $\Delta J= \pm 2$ transitions. The rapid decrease of the signal versus the probe delay $\tau_{d}$ is mainly due to the dispersion of the wave packet that occurs in asymmetric top molecules for the reasons explained in Section II. Indeed, the collisional effects included in the simulation through the application of an exponential decay factor only reduces the alignment signal at $\tau_{d}=40 \mathrm{ps}$ by $30 \%$ compared to $\tau_{d} \gtrsim 0$ ps [48]. The relatively good agreement between experiment and theory shows that the rigid molecule assumed in the model of Sec. II is a good approximation at low laser intensity.

Increasing the pump intensity leads to larger molecular alignments. It has been shown that the alignment of linear molecules results in a modification of the structural shape of the transients that is well reproduced by the theory and therefore can be used to quantify the degree of molecular alignment [14, 37]. As shown in Fig. 6, this change is also observed in ethylene as the intensity is increased from low to moderate values. In particular, we observe a net modification of the transients (indicated by vertical arrows in Fig. 6) with an asymmetry reversal occurring between $2 \times 10^{12}$ W.cm ${ }^{-2}$ and $16 \times 10^{12}$ W.cm ${ }^{-2}$, for the first $C$-type transient $\left(\tau_{d} \approx 10 \mathrm{ps}\right)$, and between $16 \times 10^{12}$ W.cm ${ }^{-2}$ and $30 \times 10^{12}$ W.cm ${ }^{-2}$, for the second $J$-type transient $\left(\tau_{d} \approx 18 \mathrm{ps}\right)$, respectively. For the in-
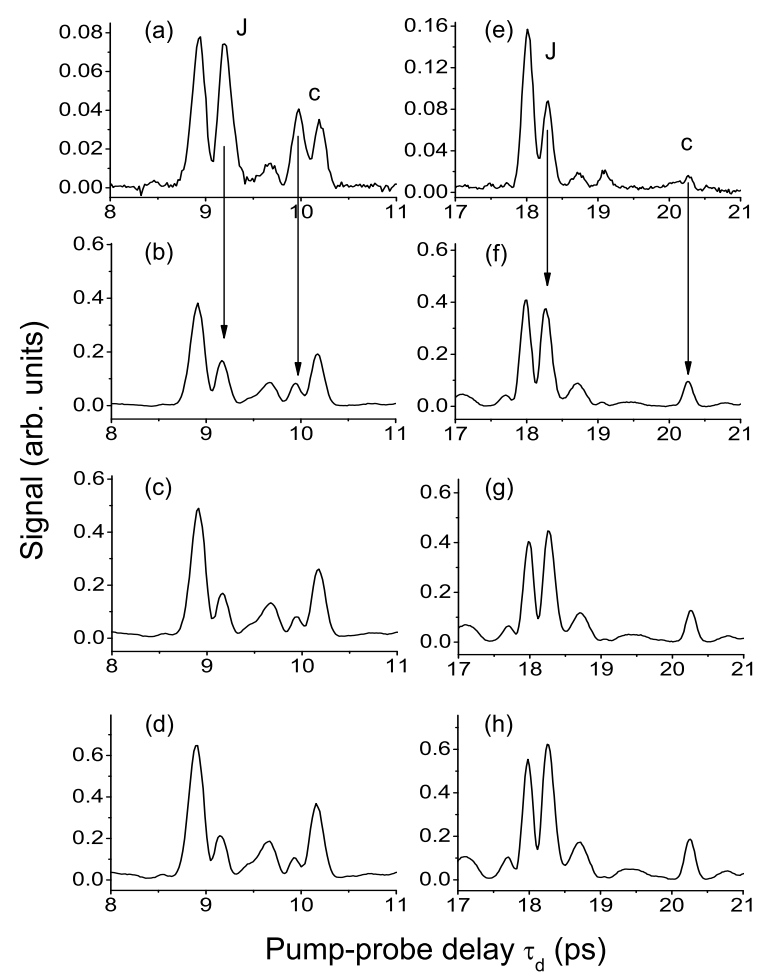

FIG. 6: Probe signals recorded in ethylene exposed to different intensities of the alignment laser: (a, e) $2 \times 10^{12} \mathrm{~W} \cdot \mathrm{cm}^{-2}$, (b, f) $16 \times 10^{12}$ W.cm ${ }^{-2},(c, g) 30 \times 10^{12}$ W.cm ${ }^{-2},(d, h)$ $50 \times 10^{12} \mathrm{~W} \cdot \mathrm{cm}^{-2}$. Only the temporal windows that correspond to the first (a-d) and second (e-h) rotational revivals of Fig. 5 are shown.

vestigated intensities, the modification of the transient shape is mainly due to the permanent alignment. As the intensity is raised, the permanent alignment and so the baseline of $\left\langle\cos ^{2} \theta\right\rangle$ increases, as shown previously in Fig. 3. Assuming that under our experimental conditions the convolution with the probe field in Eq. (8) rather affects the width than the amplitude of the transients, the measured signal can be approximated by $\left(\left\langle\cos ^{2} \theta\right\rangle-1 / 3\right)^{2}$. The permanent alignment results therefore in a larger signal when the molecular axis is aligned $\left(\left\langle\cos ^{2} \theta\right\rangle>1 / 3\right)$ compared with when it is delocalized $\left(\left\langle\cos ^{2} \theta\right\rangle<1 / 3\right)$. Considering this, we can identify from the first revival represented in Fig. 6 that the $\mathrm{C}=\mathrm{C}$ bound axis of ethylene is aligned prior to delocalized, for the $J$-type, and the opposite, for the $C$-type. This sequence alternates for the $J$-type from one to the other revivals, as verified for the second revival shown in the right side of the same figure. The modification of the transients versus intensity is also observed in the numerical simulations of Eq. (9) shown with full lines in Fig. 7. The calculated signals well reproduce the observations of Fig. 6 in what concerns the structural shape of the transients of the first revival. Concerning the second revival, 

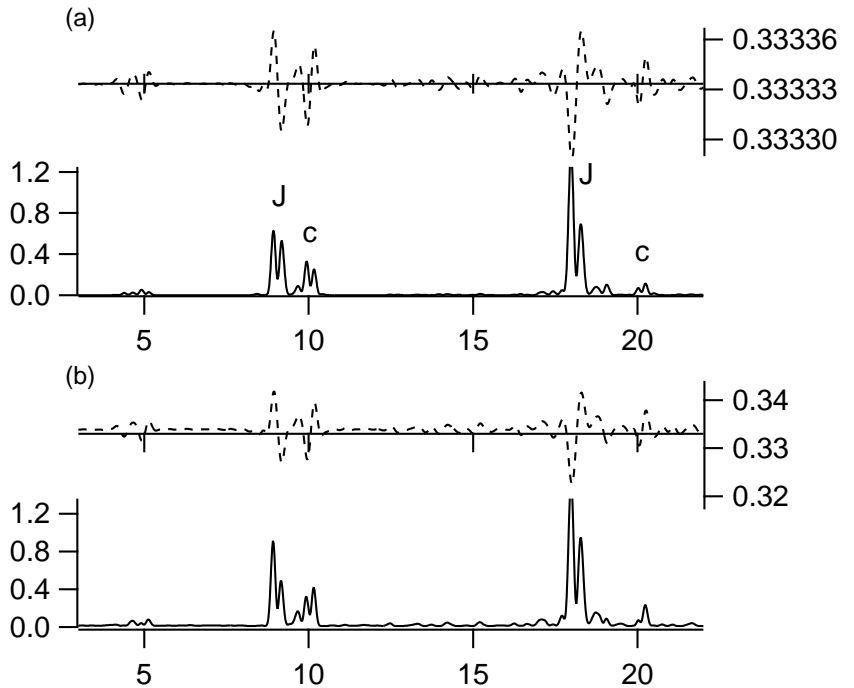

(c)

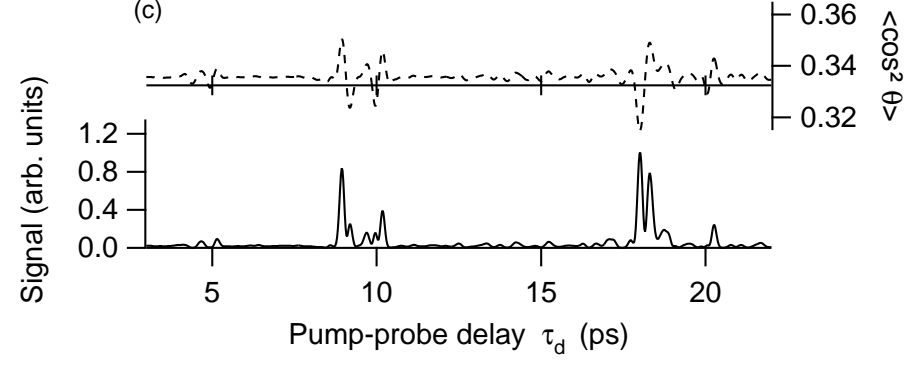

FIG. 7: Probe signal calculated for a distribution of ethylene molecules at an equilibrium temperature of $293 \mathrm{~K}$ and at different peak intensities of the alignment laser (full lines): (a) $2 \times 10^{12}$ W.cm ${ }^{-2}$, (b) $25 \times 10^{12}$ W.cm ${ }^{-2}$, (c) $50 \times 10^{12}$ W.cm ${ }^{-2}$. The corresponding values of $\left\langle\cos ^{2} \theta\right\rangle$ are depicted on the right line scale (dashed lines).

a slight disagreement is found for the $J$-type for which the theory predicts an asymmetry reversal at a larger intensity than the investigated one. This can be due to the rigid rotor model on which is based the calculations and whose approximation is less accurate at high intensities, as the laser field populates higher lying rotational levels (see for instance Fig. 8). The temporal spreading of the wave packet resulting from nonrigid molecules is expected to be more pronounced with time and therefore could support the disagreement observed at larger delays. The relative amplitudes between the first and second revival also well agree with the observations. The difference of amplitude between the two revival diminishes with the intensity. The maximum signal is reached at the second revival, the third and subsequent revivals (not shown) being of smaller amplitude. From the corresponding $\left\langle\cos ^{2} \theta\right\rangle$ values, shown with dashed lines in the same figure, we observe that the difference of signal amplitude between the two revivals is due to a permanent alignment effect and that the two first revivals produce in fact the same degree of alignment. Inspection of the $\left\langle\cos ^{2} \theta\right\rangle$ curves calculated at different intensities also stresses that the modification of the transient shapes observed in the experiment is mainly due to the change of permanent alignment. Finally, we notice from Fig. 7 a structure around 5 ps that is also observed in Fig. 5 and in the experiments performed at high intensity. It could be attributed to an hybrid transient corresponding to a pseudo-period $T_{h}=1 /(2 A-B-C)=4.3$ ps.

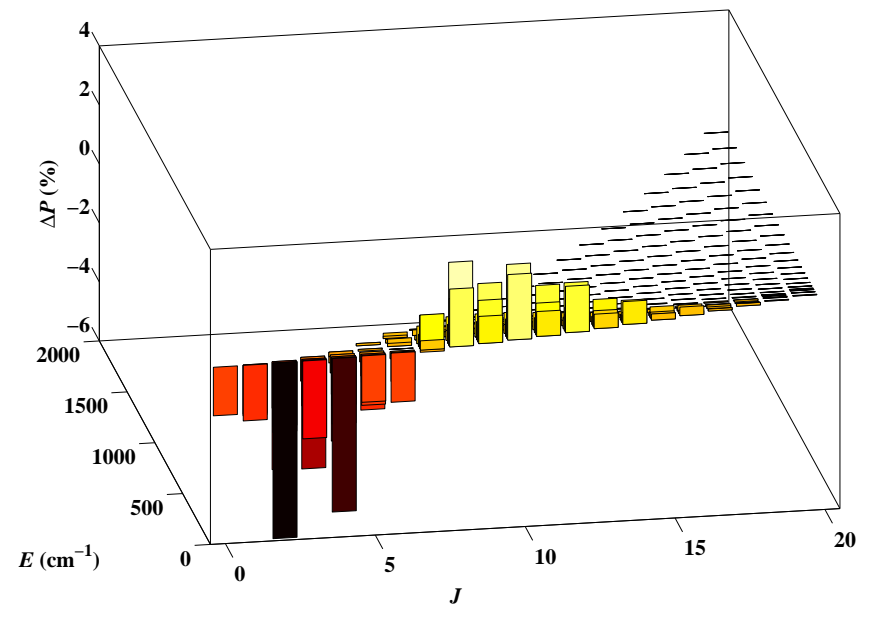

FIG. 8: (Color online) Difference between the population after and before (i.e. at thermal equilibrium) alignment for each rotational level and as a function of $J$ at $50 \mathrm{~K}$ with a peak intensity of $\mathcal{I}_{p}=50 \times 10^{12}$ W.cm ${ }^{-2}$.

The relatively weak alignment, $\max _{t}\left\langle\cos ^{2} \theta\right\rangle=0.35$ achieved at averaged intensity of $50 \times 10^{12} \mathrm{~W} . \mathrm{cm}^{-2}$ [Fig. 7 (c)], results from the large temperature of the gas sample combined with a laser intensity limited by the onset of ionization occurring in ethylene around a peak intensity of $80 \times 10^{12} \mathrm{~W} . \mathrm{cm}^{-2}$ [40]. We mention that the results of Fig. 7 have been obtained using in the calculation peak intensities comparable with averaged intensities measured during experiments. A better modeling would require taking into account the spatial distribution of intensities within the laser profiles [41]. Although integration of the laser profile in the simulation affects the transients, it does not introduce drastic changes on their respective shapes because of the relatively high temperatures used in the experiments where fine details of the transient pattern are washed out through thermal averaging.

The last point that we would like to address concerns the amplitude ratio of the $J$ - to $C$-type transients. We observe from Fig. 6, especially on the second revival, a modification of this ratio well reproduced by the simulation of Fig. 7. It shows that the intensity favors alignment preferentially for the $C$-type. A first explanation of this feature can be obtained from the numerics by analyzing the population transfers produced by the aligning laser. Figure 8 shows the population difference $\Delta P$ produced by the alignment pulse. To evidence the population transfer, the simulations are done at $50 \mathrm{~K}$. Inspection of this graph 
shows that the laser field transfers the population of the system towards the higher $J$ levels essentially along the lower part of the spectrum $|J, \tau \approx-J, M\rangle$.
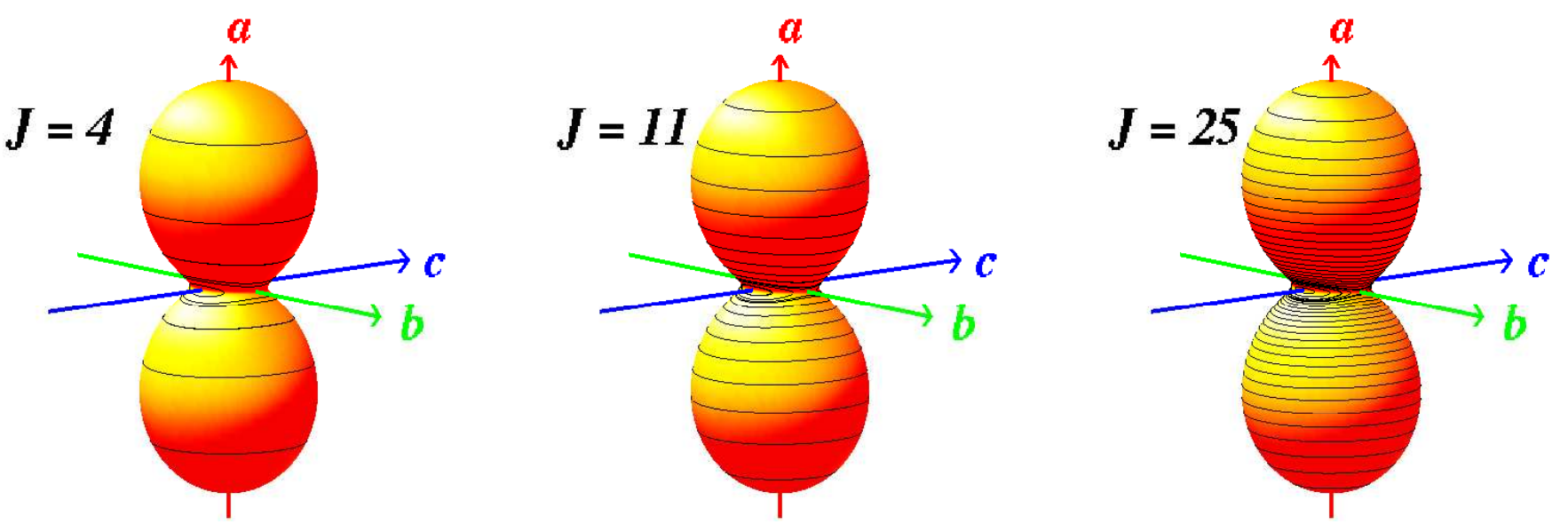

FIG. 9: (Color online) Rotational energy surface (see text) for different $J$ values. The black lines correspond to the quantum levels.

If we consider the angular momentum components as classical continuous quantities and use spherical coordinates,

$$
\begin{aligned}
& J_{x}=\sqrt{J(J+1)} \sin \Theta \cos \Phi, \\
& J_{y}=\sqrt{J(J+1)} \sin \Theta \sin \Phi, \\
& J_{z}=\sqrt{J(J+1)} \cos \Theta,
\end{aligned}
$$

then the Hamiltonian (3) defines a so-called rotational energy surface (RES) [42, 43],

$$
E_{J}(\Theta, \Phi)=E_{0}\left(J_{x}, J_{y}, J_{z}\right),
$$

for each amplitude $J$. For an asymmetric rigid rotor, points on the $a, b$ and $c$ axes are always maxima, saddle points and minima, respectively [42]. Figure 9 shows ethylene's RES for three values of $J$. This figure also shows the quantum levels as iso-energy lines on the RES that can be viewed as classical trajectories of the angular momentum vector. It can be seen that, as $J$ increases, the minima around the $c$ axis deepen and thus the number of low energy levels for which the molecule is trapped in these troughs increases. Classically, they correspond to closed trajectories around the $c$ axis. We can thus understand that if the population is shifted toward higher $J$ values as in Figure 8, the $C$-type transients increase. In any case, there are also levels above the barrier between the two minima of the $c$-axis (i.e. trajectories going through or near $b$ ) which are populated. They correspond to an angular momentum vector situated near the $b c$ equatorial plane of the RES leading to $J$-type transients (i.e. the molecule "sees" a mean rotational constant $(B+C) / 2$; in the case of a prolate symmetric top,
$B=C$ and the whole equatorial circle is a minimum, thus only $J$-type transients exist). For a given $J$ value, higher energy levels which correspond to closed classical trajectories around the $a$ axis would lead to $A$-type transients, but as these levels are not populated under the conditions studied here, they are not observed. The result of the field alignment is therefore a free rotation of the molecule which is more pronounced in the molecular plane. In ethylene at room temperature, this effect is relatively modest and even for the largest intensities the $J$-type remains larger than the $C$-type transients. In the alignment of iodiobenzene [15], the experiments performed at low temperature show that the $C$-type transients are the most pronounced. A second explanation refers to a classical argument. The result of the laser interaction is that the induced molecular dipole is moved towards the $\mathrm{C}=\mathrm{C}$ bound axis of the molecule, as the former approaches the field direction. In the same time, the molecule rotates towards a position in which the molecular plane contains the field direction (see Sec. II). Since the torque applied by the field to the molecule is perpendicular to both field and induced dipole, its direction becomes more localized perpendicular to the molecular plane as the field increases. This produces a rotation of the molecule about the $c$-axis that favors the $C$-types transients after the pulse turn off. 


\section{CONCLUSION}

Field-free one-dimensional alignment of a near symmetric top molecule has been investigated in ethylene. The experiments have been conducted using short linearly polarized laser pulses to produce and probe the molecular alignment produced in a gas cell. The probe process has been performed by recording the transient birefringence of the gas sample resulting from the molecular alignment. The optical measurements have been interpreted by the use of a theoretical model that is based on the simulation of the Schrödinger equation for the non-resonant interaction of a short pulse with an asymmetric top molecule. In this model, we describe the alignment by considering the expectation values of the direction cosines with respect to the field direction. Their temporal evolutions show that the molecule undergoes a predominant alignment along its major principal axis of polarizability (i.e. the $\mathrm{C}=\mathrm{C}$ bound axis, in ethylene) but also a fast alternation of moderate alignments along the two other axes. Inspection of the angular distribution calculated at different times reveals that while the major axis is aligned (resp. delocalized) the other axes are successively delocalized (resp. aligned).

The first experiment is conducted in the weak field regime. The probe signal shows rotational revivals with identified $J$-type and $C$-type transients, the former being of largest amplitude. Each transient results from alignment and planar delocalization of the $\mathrm{C}=\mathrm{C}$ bound axis of ethylene that is well reproduced by the simulation. The intensity dependence of the probe signal is then investigated. A clear modification of the transients shape with intensity, resulting essentially from the creation of a per- manent alignment, is observed in agreement with the theory. The increasing amplitude of the $C$-type transients with the degree of alignment suggests that the laser field rotates the molecule preferentially about the $c$ axis, i.e within the molecular plane of ethylene. This is supported by an analysis of the populations transfered by the laser field during the pulse and the repartition of the quantum levels of the molecule on the rotational energy surface.

The alignment efficiency is limited by the intensity that the molecule can stand until it ionizes. The relatively small anisotropy of polarizability of ethylene $\left(\Delta \alpha_{z y}=1.7 \AA^{3}\right)$, if one compare to other asymmetric top molecules of $\mathrm{C}_{2 v}$-type like for instance iodobenzene $\left(\Delta \alpha_{z y}=10.8 \AA^{3}\right)$ or iodopentafluorbenzene $\left(\Delta \alpha_{z y}=\right.$ $11.0 \AA^{3}$ ) [44], combined with the large number of rotational levels populated at room temperature makes the molecule difficult to align. Improvement will be possible by (i) lowering the rotational temperature with for instance a supersonic gas expansion [49], (ii) using shape pulse techniques to optimize and control the process.

Ethylene molecules present a particular interest in the context of adsorption on nanoporous solids such as zeolites $[45,46]$. The huge electric fields arising inside the cavities of such crystals could lead to interesting alignment phenomena.

\section{Acknowledgments}

This work was supported by the Conseil Régional de Bourgogne, the Action Concertée Incitative Photonique from the French Ministry of Research, and a Marie Curie European Reintegration Grant within the 6th European Community RTD Framework Program.
[1] H. Stapelfeldt and T. Seideman, Rev. Mod. Phys. 75, 543 (2003).

[2] J. Itatani, D. Zeidler, J. Levesque, M. Spanner, D. M. Villeneuve, and P. B. Corkum, Phys. Rev. lett. 94, 123902 (2005).

[3] X. Zhou, X. M. Tong, Z. X. Zhao, and C. D. Lin, Phys. Rev. A 72, 033412 (2005).

[4] D. Zeidler, A. Staudte, A. B. Bardon, D. M. Villeneuve, R. Dorner, and P. B. Corkum, Phys. Rev. Lett. 95, 203003 (2005)

[5] J. J. Larsen, H. Sakai, C. P. Safvan, I. Wendt-Larsen, and H. Stapelfeldt, J. Chem. Phys. 111, 7774 (1999).

[6] D. Normand, L. A. Lompre, and C. Cornaggia, J. Phys. B 25, L497 (1992).

[7] J. Ortigoso, M. Rodriguez, M. Gupta, and B. Friedrich, J. Chem. Phys. 110, 3870 (1999).

[8] B. Friedrich and D. Herschbach, Phys. Rev. Lett. 74, 4623 (1995).

[9] K. Hoshina, K. Yamanouchi, T. Ohshima, Y. Ose, and H. Todokoro, J. Chem. Phys. 118, 6211 (2003).

[10] T. Seideman, J. Chem. Phys. 115, 5965 (2001).

[11] F. Rosca-Pruna and M. J. J. Vrakking, Phys. Rev. Lett. 87, 153902 (2001).
[12] F. Rosca-Pruna and M. J. J. Vrakking, J. Chem. Phys. 116, 6567 (2002).

[13] F. Rosca-Pruna and M. J. J. Vrakking, J. Chem. Phys. 116, 6579 (2002).

[14] V. Renard, M. Renard, S. Guérin, Y. T. Pashayan, B. Lavorel, O. Faucher, and H. R. Jauslin, Phys. Rev. Lett. 90, 153601 (2003).

[15] E. Péronne, M. D. Poulsen, C. Z. Bisgaard, H. Stapelfeldt, and T. Seideman, Phys. Rev. Lett. 91, 043003 (2003).

[16] R. Torres, R. de Nalda, and J. P. Marangos, Phys. Rev. A 72, 023420 (2005).

[17] J. G. Underwood, M. Spanner, M. Y. Ivanov, J. Mottershead, B. J. Sussman, and A. Stolow, Phys. Rev. Lett. 90, 223001 (2003).

[18] I. S. Averbukh and R. Arvieu, Phys. Rev. Lett. 87, 163601 (2001).

[19] M. Leibscher, I. S. Averbukh, and H. Rabitz, Phys. Rev. Lett. 90, 213001 (2003).

[20] M. Leibscher, I. S. Averbukh, and H. Rabitz, Phys. Rev. A 69, 013402 (2004).

[21] K. F. Lee, I. V. Litvinyuk, P. W. Dooley, M. Spanner, D. M. Villeneuve, and P. B. Corkum, J. Phys. B 37, L43 
(2004).

[22] C. Z. Bisgaard, M. D. Poulsen, E. Péronne, S. S. Viftrup, and H. Stapelfeldt, Phys. Rev. Lett. 92, 173004 (2004).

[23] M. Spanner, E. A. Shapiro, and M. Ivanov, Phys. Rev. Lett. 92, 093001 (2004).

[24] K. F. Lee, D. M. Villeneuve, P. B. Corkum, and E. A. Shapiro, Phys. Rev. Lett. 93, 233601 (2004).

[25] M. Renard, E. Hertz, S. Guerin, H. R. Jauslin, B. Lavorel, and O. Faucher, Phys. Rev. A 72, 025401 (2005).

[26] T. Seideman, J. Chem. Phys. 106, 2881 (1997).

[27] D. Daems, S. Guerin, E. Hertz, H. R. Jauslin, B. Lavorel, and O. Faucher, Phys. Rev. Lett. 95, 063005 (2005).

[28] E. Hamilton, T. Seideman, T. Ejdrup, M. D. Poulsen, C. Z. Bisgaard, S. S. Viftrup, and H. Stapelfeldt, Phys. Rev. A 72, 043402 (2005).

[29] J. J. Larsen, K. Hald, N. Bjerre, H. Stapelfeldt, and T. Seideman, Phys. Rev. Lett. 85, 2470 (2000).

[30] J. G. Underwood, B. J. Sussman, and A. Stolow, Phys. Rev. Lett. 94, 143002 (2005).

[31] M. D. Poulsen, E. Péronne, H. Stapelfeldt, C. Z. Bisgaard, S. S. Viftrup, E. Hamilton, and T. Seideman, J. Chem. Phys. 121, 783 (2004).

[32] R. N. Zare, Angular Momentum (Wiley, New York, 1988).

[33] D. Papoušek and M. R. Aliev, Molecular VibrationalRotational Spectra, Studies in Physical and Theoretical Chemistry (Elsevier, Amsterdam - Oxford - New York, 1982).

[34] T. L. Tan, S. Y. Lau, P. P. Ong, K. L. Goh, and H. H. Teo, J. Mol. Spectrosc. 203, 310 (2000).

[35] A. J. Russel and M. A. Spackman, Mol. Phys. 98, 855 (2000).
[36] T. Seideman, Phys. Rev. Lett. 83, 4971 (1999).

[37] V. Renard, M. Renard, A. Rouzée, S. Guérin, H. R. Jauslin, B. Lavorel, and O. Faucher, Phys. Rev. A 70, 33420 (2004).

[38] P. M. Felker and A. H. Zewail, in Femtosecond Chemistry, edited by J. Manz and W. L. (VCH, Weinheim, New York, 1995), vol. 1, p. 193.

[39] H. M. Frey, P. Beaud, T. Gerber, B. Mischler, P. P. Radi, and A. P. Tzannis, J. Raman Spectrosc. 31, 71 (2000).

[40] T. K. Kjeldsen, C. Z. Bisgaard, L. B. Madsen, and H. Stapelfeldt, Phys. Rev. A 71, 013418 (2005).

[41] A. Rouzée, V. Renard, B. Lavorel, and O. Faucher, J. Phys. B 38, 2329 (2005).

[42] W. G. Harter, Comp. Phys. Rep. 8, 319 (1988).

[43] G. Dhont, D. Sadovskii, B. Zhilinskii, and V. Boudon, J. Mol. Spectrosc. 201, 95 (2000).

[44] M. D. Poulsen, Ph.D. thesis, University of Aarhus, Denmark (2005).

[45] J. Limtrakul, T. Nanok, S. Jungsuttiwong, P. Khongpracha, and T. N. Truong, Chem. Phys. Lett. 349, 161 (2001).

[46] V. Bernardet, A. Decrette, J. M. Simon, O. Bertrand, G. Weber, and J. P. Bellat, Mol. Phys. 102, 1859 (2004).

[47] L. S. Rothman, A. Barbe, D. C. Benner, L. R. Brown, C. Camy Peyret, M. R. Carleer, K. Chance, C. Clerbaux, V. Dana, V. M. Devi, et al., JQSRT 82, 5 (2003).

[48] In our experimental conditions, the homogeneous linewidth of ethylene is estimated about $1.35 \times$ $10^{9} \mathrm{~Hz}$ [47].

[49] Under investigation. 